\title{
Diabetes With Co-Morbidities Of Covid19 Using Covid19 Lungs Images
}

\section{Vadthe Narasimha, Dr.M. Dhanalakshmi}

\author{
Research Scholar in JNTUH, Assistant Professor, CSE Dept, CMR College of Engineering \& Technology \\ Hyderabad, India. \\ Chinna.narasimha63@gmail.com \\ Professor, Dept of Information Technology, JNTUH Jagityala, Hyderabad. \\ Dhana.miryala@gmail.com
}

Article History:Received:11 January 2021; Accepted: 27 February 2021; Published online: 5 April 2021

\begin{abstract}
Diabetes is a nightmare disease to fight, and it's prevalence of diabetes is been increasing every single day worldwide, India seems recording a high number of cases. As a condition, diabetes can also invite other health problems, COVID-19 being one of them. COVID-19 that's reputed to have emerged out of Wuhan (China) has generated a severe effect on nearly each modern society of the planet. As a result of issues due to this specific wellness catastrophe all around the Earth, that the World Health Organisation (WHO) has announced it's a international outbreak. COVID-19 is still a state that will get worse with co-morbidities. Researches snare that individuals Afflicted by diabetes Experience with worse results, possess a higher Danger of seriousness, and extended recovery period. In this paper we are trying to study and analyse the impact of covid-19 on the diabetic patients using the lung chest images. This study helps the doctors to understand more about the patients and overcome serious factors. We use Deep Learning methods to study the images and co-morbidities of diabetics with covid-19.
\end{abstract}

Keywords: Diabetes, Image segmentation, COVID-19, Lungs, Deep Learning.

\section{INTRODUCTION}

For first time inside this century, the entire world is currently confronting a health disaster which has influenced every individual being on this world somehow or the other. However on positive aspect, the scale of COVID-19 has invented greater collaboration amongst countries. We're learning from eachother and seeking to comprise this dreaded disorder until it promises lives. But, it Is a Fact It Has interrupted our own lives just like never before. Coronavirus disease 2019, or COVID-19, is caused by severe acute respiratory syndrome coronavirus 2 (SARSCoV-2). It was first identified in Wuhan, China in 2019 and rapidly spread across the world such that it was declared a pandemic by the World Health Organization on March 11, 2020. COVID-19 can rapidly spread from person to person, through respiratory droplets produced through breathing, singing, talking, coughing, or sneezing. COVID-19 continues to be spread in the community in multiple states, prompting reversal of reopening plans.

In medicinal sciences, comorbidity has characterized like a state found simultaneously; yet, people by having an extra illness or some related health illness. In short, co-morbidity defines the consequence of the other cases a single patient may possibly have other compared to the key state of attention and certainly will be psychological or physiological. American epidemiologist A. R. Feinstein initial released the word co-morbidity from the $1970 \mathrm{~s}$. The mixture of the protracted disorder for example diabetes and also a significant viral disease such as COVID-19 provides challenging struggle for the health vocation to rescue. Both equally diabetes type 1 and two really are a family group of ailments which ends in elevated glucose amount at the bloodstream circulation. After your human body struggling to generate a sufficient quantity hormone insulin, which enables your entire body to acquire sugar into cells to get energy, then the blood sugar accumulates in blood vessels. The total diabetes phenomenon at 20-19 estimated to become $9.3 \%$ having a likely worth of 463 million folks afflicted by the status. Patients having diabetes believed high-risk patients to get infections that were obtained. The more complicated the sugar, the higher the more complex the possibility of ailments. Moreover, most individuals having diabetes grow more than co-morbidities that boost this kind of pitfalls. Diabetes diabetes, cardiovascular disease (CVD) and Weight Problems have been couple common or known ailments Associated with metabolic syndrome, and entirely or independently, They Are Sometimes prone to a group of triggers connected to COVID-19 pathogenesis.

As stated by the American Diabetes Association (ADA), in the moment, there are inadequate information to reveal if people who have diabetes are somewhat more inclined to turn into infected using COVID-19nonetheless, people who have diabetes have significantly more favorable consequences, for example as elevated degrees of acute 
ailments. It's understood that elderly adults and Those That Have chronic obstructive pulmonary disorder, Cardiovascular Disease, diabetes, obesity, chronic kidney disorder diabetes and obesity are all in increased risk for severe COVID-19 requiring hospitalization.5 With a long incubation time reported to be up to 24 days and both symptomatic and asymptomatic carriers able to transmit the disease, COVID-19 has proven to be highly infectious. This report critiques the existent literature about the function of diabetes for a risk variable for SARS-CoV-2 illness and consequences, together with reference into this statistics regarding the incidence of diabetes one of COVID-19 sufferers and its particular own association together with all the seriousness of this disorder. What's More, the Potential mechanics linking diabetes into COVID-19 Is Going to Be discussed, having a Concentration on the Use of diabetes-associated comorbidities/complications.

\section{Related Work}

Primarily, reasonably tiny scientific tests from Wuhan along with also the Hubei state and from other Oriental regions demonstrate varying incidence rates of diabetes among individuals hospitalized together using COVID-19, together with differences probable representing the era of this samplethe hospital surroundings, and also the geographic location. In 2 multicenter, nationally reviews, diabetes has been within 7.4\% of 1099 (median age 4-7 years) [inch ] as well as at $8.2 \%$ of inch, 590 (average era, 48.9 several many a long time ) [two ] hospitalized folks. Moreover, of 7337 men confessed to nineteen hospitals at the Hubei state (median age 54 years), 952 (13.0\% ) had type two diabetes, where as a record of this Chinese Center for Disease Control and Prevention (China CDC), that nevertheless contained additionally non-hospitalized folks, revealed a decrease incidence of diabetes $(5.3 \%)$ one of 44,672 supported COVID-19 instances by way of February 11," 20 20. A number of meta-analyses for example Chinese COVID-19 sufferers affirmed an incidence of diabetes approximately 8--10\%, i.e., perhaps maybe not larger, in case any such thing, than that at general people, that had been $12.9 \%$ in 2013 amid individuals aged 40--59 decades ago Moreover, the incidence was $11.2 \%$ (9.8\% after correcting to heterogeneity) at a meta analysis as well as two scientific research by the united states plus a study by France as well as large as $23.8 \%$ in a second meta analysis by which significantly a lot more than $1 / 2$ patients were out of an US examine.

Viewing studies from out China, a single-center analyze by Padua, Italy, revealed that one of 146 hospitalized people (average age 65.3 a long time ), the incidence of diabetes has been $8.9 \%$, all over once more higher than at the coeval basic people by an identical area $(11.0 \%)$. The other single-center Italian poll out of Milan documented that a melancholy incidence of $14.9 \%$ one of 410 hospitalized folks who have COVID-19 (median age 65 years). Prevalence levels were higher in US individuals hospitalized using COVID-19, which range from 22.6 to $37.2 \%$. These statistics are a lot more compared to those claimed at the typical US, i.e., $13.0 \%$, and higher than those detected one of US folks aged 45--64 decades, i.e., $17.5 \%$, nevertheless at many studies that the median age has been near for the top limit with this scope.

Seeing polls from out China, a single-center analyze by Padua, Italy, revealed that one of 146 hospitalized people (average age 65.3 a long time ), the incidence of diabetes has been $8.9 \%$, all over once more higher than at the coeval basic people by an identical area $(11.0 \%)$. The other single-center Italian poll out of Milan documented that a melancholy incidence of $14.9 \%$ one of 410 hospitalized folks who have COVID-19 (median age 65 years). Remarkably, the incidence of diabetes has been high one of 1339 COVID-19 in seven associations at Madrid, Spain (average age 69.1 several many a long time ), when compared using 13,390 matched controllers $(27.2 \%$ versus 20.3 $\%$; primitive odds ratio, OR, respectively 1.50 [95\% confidence interval, CI, 1.30--1.73]). Prevalence levels were higher in US individuals hospitalized using COVID-19, which range from 22.6 to $37.2 \%$. These statistics are a lot more compared to those claimed at the typical US, i.e., $13.0 \%$, and higher than those detected one of US folks aged 45--64 decades, i.e., $17.5 \%$, nevertheless at many studies that the median age has been near for the top limit with this scope.

Absolutely, statistics from all around the planet demonstrate the incidence of diabetes amongst patients may lead to COVID-19 and it's higher than that detected from the typical populace, specially if due to the fact a large part of the existent reports have been out of hospitalized people also, thus, might possibly suffer in some selection/referral prejudice which might lead to a heightened incidence of diabetes from preventing less intense, non-hospitalized men and women. So, available statistics Indicate That diabetes Might Be a hazard variable for SARS-CoV-2 disease. 


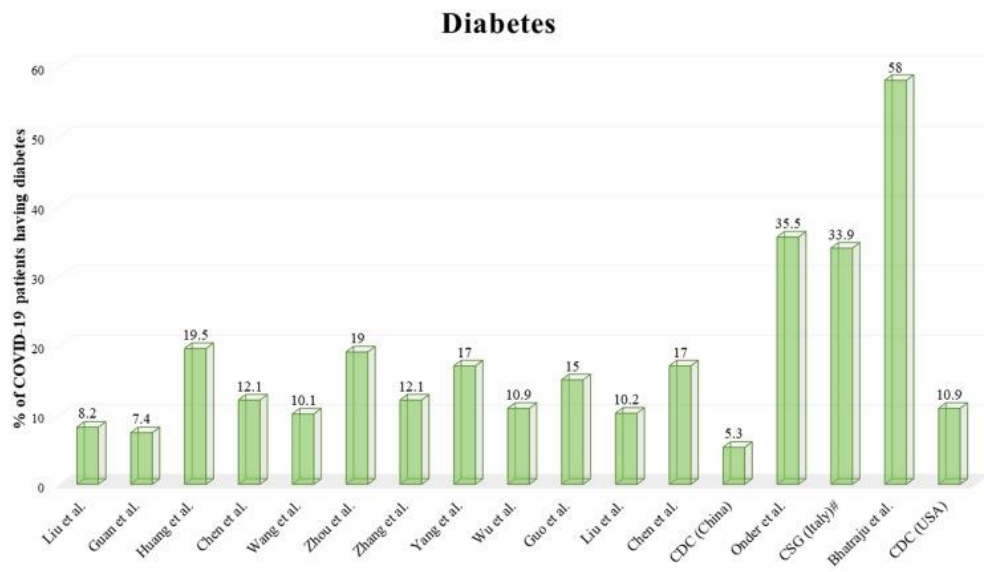

Figure 1 : percentage of COVID-19 patients having diabetes

Some Reports and meta-analyses have Analyzed the Effect of diabetes on COVID-19 Seriousness, Characterized as Desire minus no Demand for Nausea, Crucial as Opposed to Disease Disease, Development as Opposed to nonprogression, ARDS as Opposed to non-ARDS, Condition minus no Need for ICU admission or mechanical Venting, Lethal as Opposed to Celiac Disorder, or Incident versus Nonoccurrence of an Combination Effect Blending them.

\section{Diabetes and COVID-19 (SARS-CoV-2):}

It's demonstrated that individuals having diabetes along with inadequate blood sugar management possess a definite correlation between the intensity of disorder and also the danger of passing once they're afflicted with herpes such as the SARS-CoV [4], pandemic flu A (H1N1) [5], along with MERScoV [6]. Previous scientific tests ought to be contemplated and appreciated because SARS-CoV," MERScoV, and also SARS-CoV-2 are typical corona viruses. It appears that diabetics have a high susceptibility to book coronavirus and greater mortality immediately soon following disease. However some scholars have known for far superior blood sugar control in patients using COVID-19 sufferers [7], there's little statistics in the sugar of covid-19 sufferers and its particular own effect on diabetic disorders. Likewise diabetes is just one of those co morbidities related to adverse effects in elderly saysCoV-2 sufferers.

Diabetes mellitus is serious swelling. This inflammatory method will reflect the inherent mechanics causing a high susceptibility to illness, leading to a worse prognosis for most all patients. In addition, diminished elastic immunity might cause a first delay in cell-mediated resistant stimulation and following hyper-inflammatory reaction. Diabetics afflicted with SARS-CoV-2 can cause a increased stress illness, re-leasing hyperglycemic hormones like glucocorticoids and catecholamines, resulting in elevated blood sugar levels and strange versions [9]. But, it's still perhaps not too evident the amount of immune and inflammation reactions precisely happen in such people, also if hyperglycemia or bile will change the virulence of both SARS-CoV-2, also perhaps herpes itself may undoubtedly probably hinder nitric oxide or blood glucose sugar handle. It might be well worth noting the the interaction between both COVID-19 and diabetes could possibly be reciprocal disturbance. SARS-CoV-2 could worsen present diabetes, also some times could even cause ordinary sufferers to own diabetes, even its own mechanics may possibly function: angiotensin-converting receptor 2(ACE2)could be your pathway which SARS-CoV-2 input into the body. It expressed from the pancreas and also could may play a job while sufferers possess insulin resistance and insulin secretion ailments [10,11]. We assume the Possible mechanism which obesity is much significantly more susceptibility into COVID-19 possibly:

1) The immune system dysfunction accompanied with impaired, neutrophils, macrophages, B cells, T cell.

2) Decreased virus clearance.

3) Disorder of inflammatory response process.

4) Abnormal pro-inflammatory response may enhance susceptibility to cytokine storm syndrome.

5) SARS-CoV-2 may damage the pancreas.

\section{Methodology}

Clinical forms of COVID-19 had been characterized dependent on the diagnostic and therapy method principle for acute respiratory syndrome coronavirus two (SARS-CoV-2) the following: gentle variety: gentle medical symptoms minus dementia. Shared kind: Stress and respiratory ailments together using disabilities in imaging and Diabetics. 
Predicated in their own blood sugar ranges, the individuals had been assigned to three classes.

1. Even the euglycemia category: that there have been 44 sufferers written of 2-1 men and 23 girls, each one whom experienced no histories of diabetes, obesity and also the variety of these era has been 27-52 years-old.

2. The secondary hyperglycemia category: that there were 2-2 patients comprising of 17 men and five guys, that met with that the terms without a previous histories of diabetes.

3. The diabetes bunch: that there were also 14 sufferers for example 10 men and 4 guys, those were kind two diabetes mellitus individuals.

The data is collected based on the chest lung $X$ rays. Even a convolutional neural system without pre processing with diminished levels is really capable of discovering COVID-19 at a restricted variety of, also in imbalanced, torso $\mathrm{x}$ ray pictures. The process adopted to coach the neural system is clarified, together side the procedure which has been followed closely to generate the data set. The system and also the foundation code used to coach it can be obtained at https://github.com/jdariasl/COVIDNET, therefore results may be conveniently replicated by different research workers. Even a convolution neural system without pre processing with diminished levels is really capable of discovering COVID-19 at a restricted variety of, also in imbalanced, torso $\mathrm{x}$ ray pictures.

The heart of the machine can be just really actually a profound CNN depending about the COVID-Net2 suggested. Some alterations were built to comprise regularization parts from the previous two dumb layers and also a weighted categorical cross-entropy reduction work to pay to get your own course imbalance. The system arrangement has been likewise refactored to permit gradient-based localization estimations, that can be utilised after trained inside the quest to get a supplementary version.

The system had been trained with all the corpus explained utilizing the Adam optimizer having an understanding speed coverage: that the instruction speed declines when finding out stagnates for a certain time (i.e.,"'endurance'). What's more, info enhancement for glaucoma along with COVID-19 lessons was leveraged with all the subsequent enhancement kinds: flat reverse, Gaussian noise having a variance of both 0.015 , spinning, elastic deformation, and scaling. The version of this COVID-Net was created and assessed utilizing the PyTorch library. Even the CNN capabilities from every picture are concatenated with a canning performance, and also the subsequent element map has been fed directly into about a few fully attached levels to create a potential score to every single category. The very initial two entirely attached layers incorporate drop-out regularization of both 0.3 and also ReLU detection purposes. Drop-out was essential since the first network tended to overfit because the beginning of the training stage.

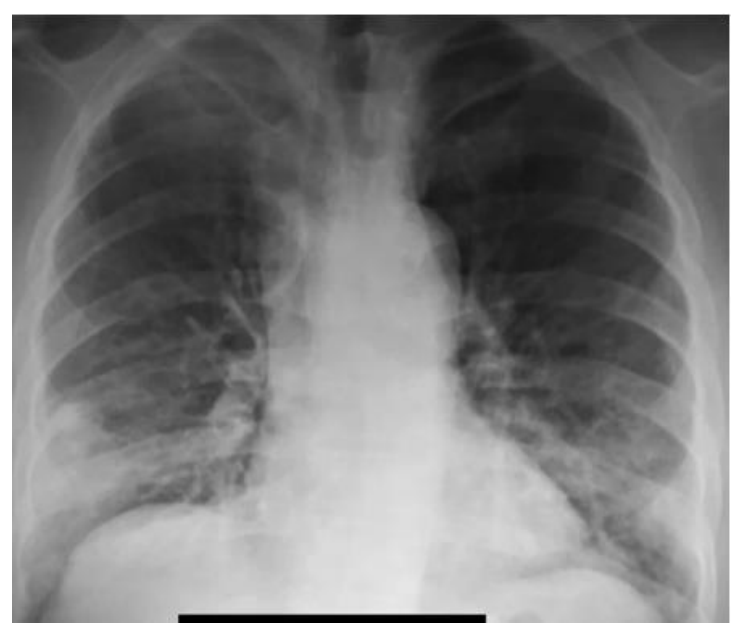

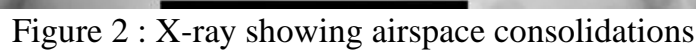




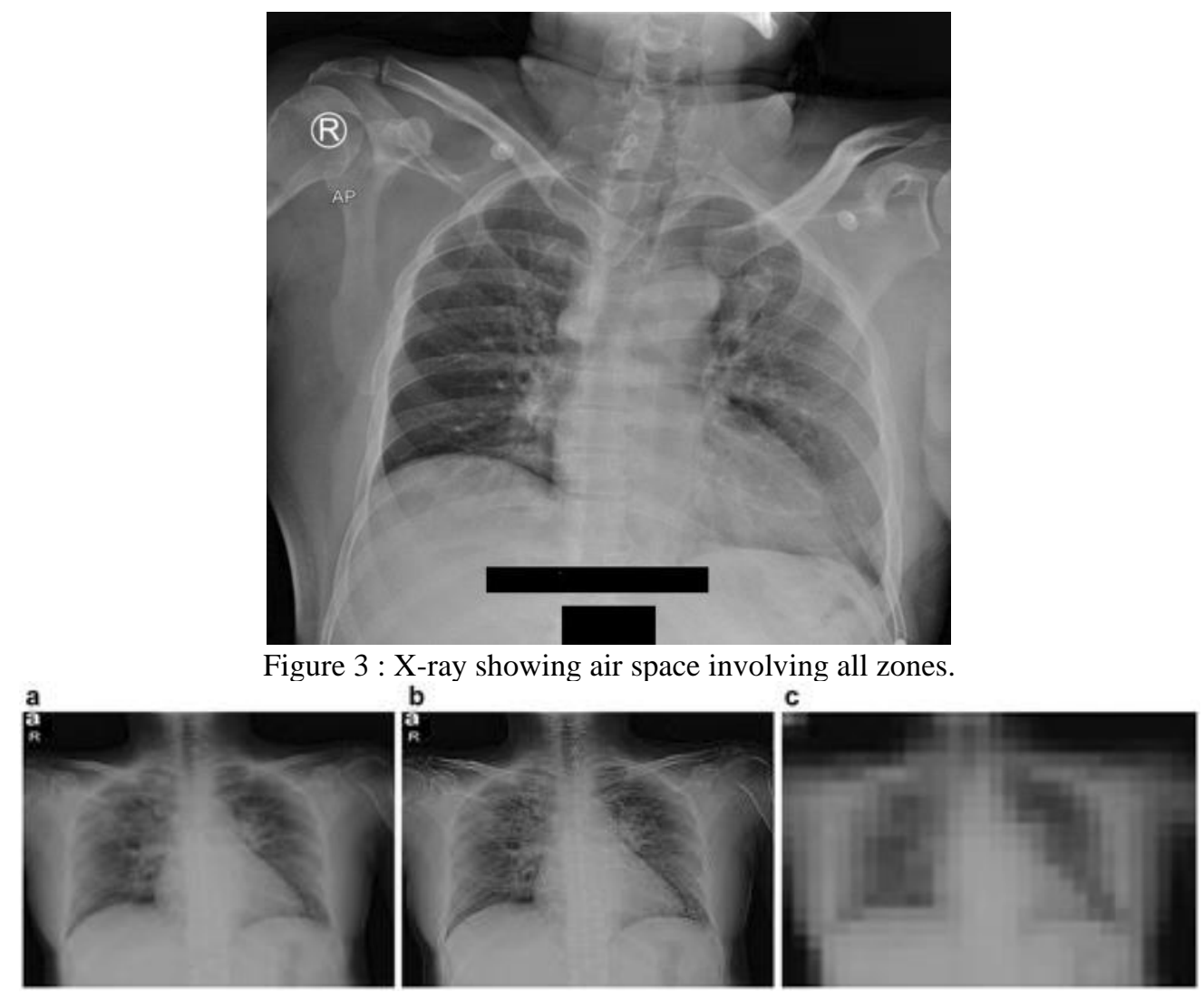

Figure 4 : Images processed with the aspect ratio with different sections.

Further, laser augmentation is put within this accumulated data-set to stop over-fitting. The augmentations contained spinning, zoomsharing and even sharing of all graphics. The info was subsequently shuffled to generalize the version and cut back over-fitting. Next, the geared up data set was usedto coach the suggested version. To get far superior investigation, about a few distinct models are executed, then their operation had been contrasted to figure out the precision. From the provided versions, we used Leaky ReLU activation rather than the at first utilised relu detection feature, making it being a publication procedure. This Procedure Will Help to Accelerate the practice plus avoids the Issue of Useless nerves.

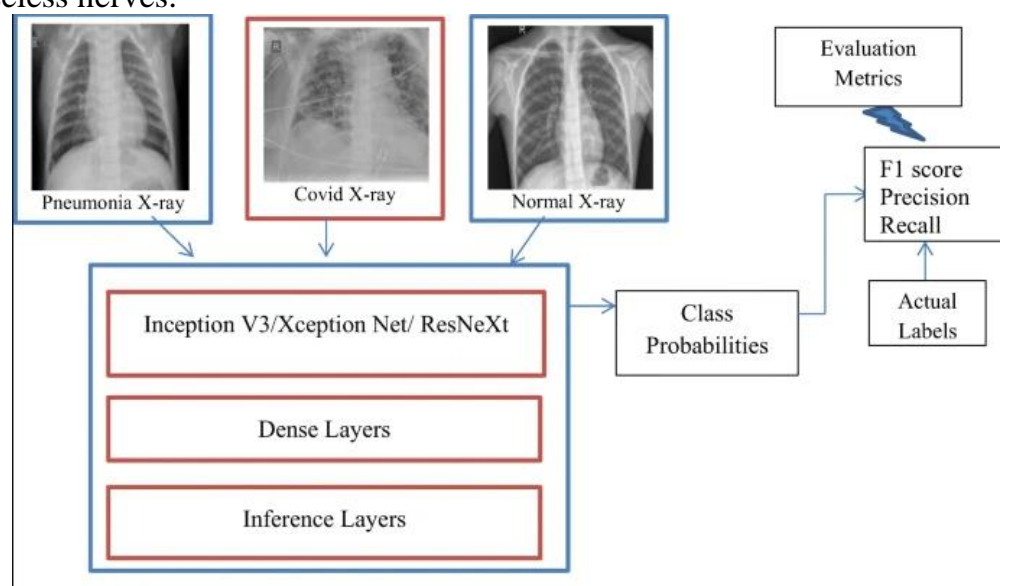

Figure 5 : Proposed model for chest X-ray dataset evaluation

With all such antecedents at heart that this newspaper employs a profound learning algorithm-based on CNN, info enhancement, along with regularization methods to manage info imbalance to its discrimination in amongst COVID19, controls, along with also different kinds of pneumonia. The processes are analyzed with probably the many intensive corpus thus far, towards the writers' comprehension. Three distinct sets of experiments had been conducted out from the quest to find its very convenient and pragmatic strategy. For the end the newspaper additionally utilizes 
explain ability methods to put on insight into the ways on the way a neural system accomplishes, along with interpretability regarding the approximate one of those parts of interest chosen from the system along with also people which tend to be more prone influenced by COVID-19. An Essential evaluation of variables that Influence the Operation of Computerized approaches predicated on profound learning can be completed.

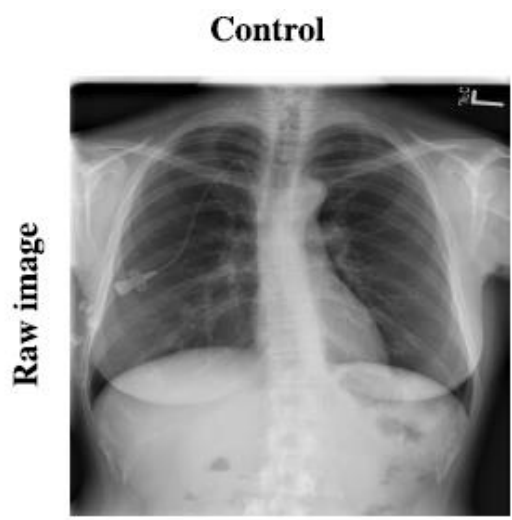

(a)
Pneumonia

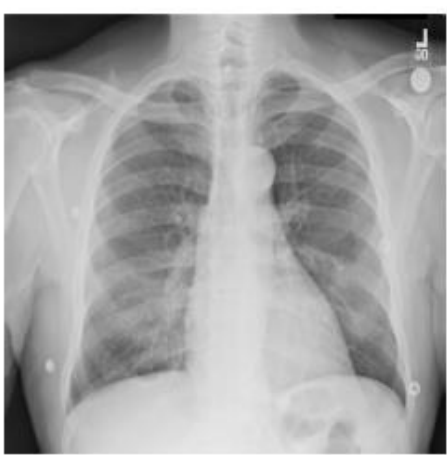

(b)
COVID-19

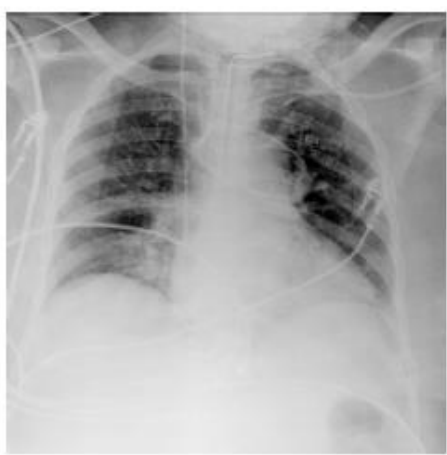

(c)

Figure 6: The original images of the patients

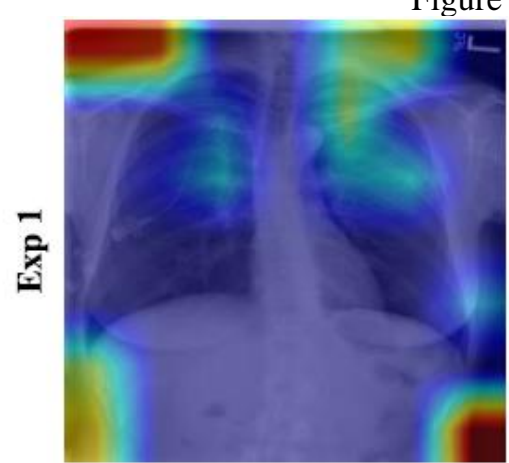

(d)

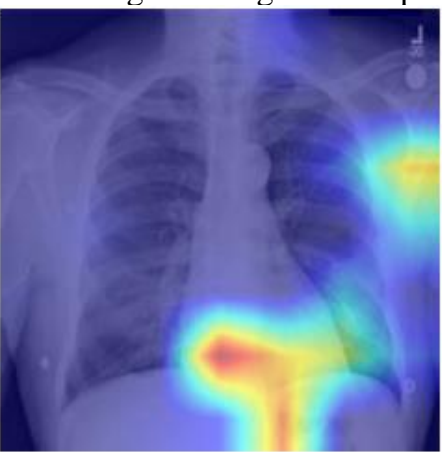

(e)

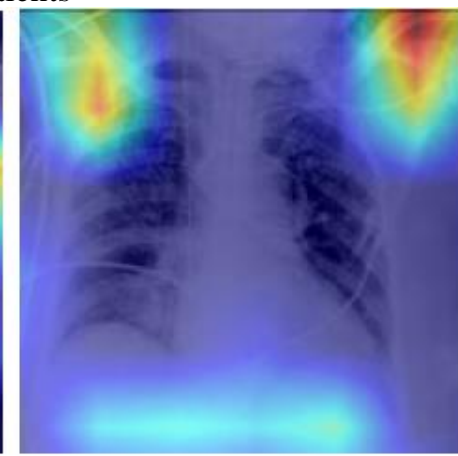

(f)

Figure 7: The images has been analyzed using the low learning rate

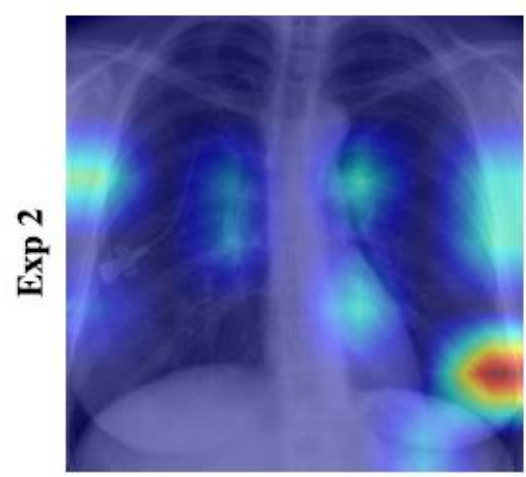

(g)

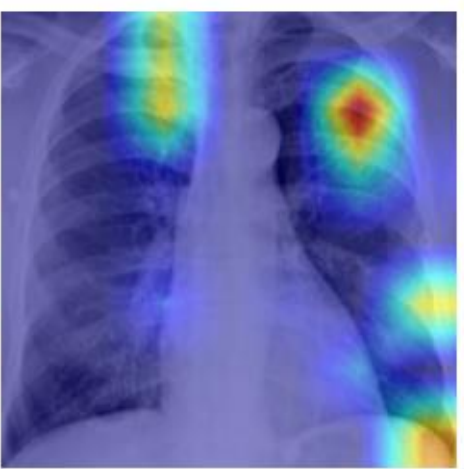

(h)

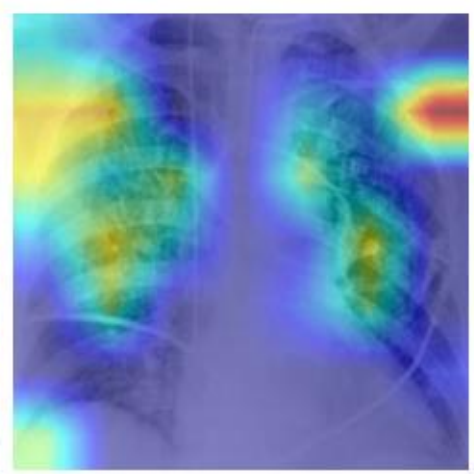

(i) 
Figure 8: The experiment is made with enough epoch values for consistency in low error rate.

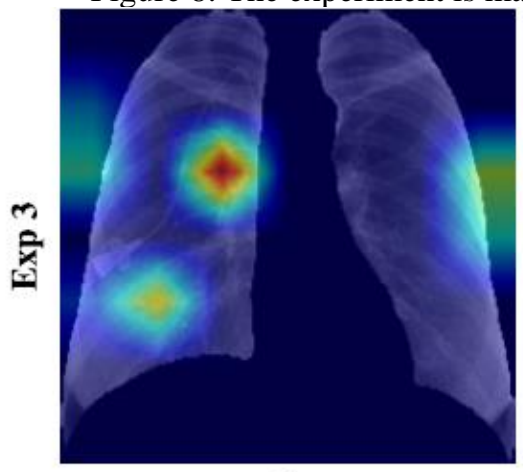

(j)

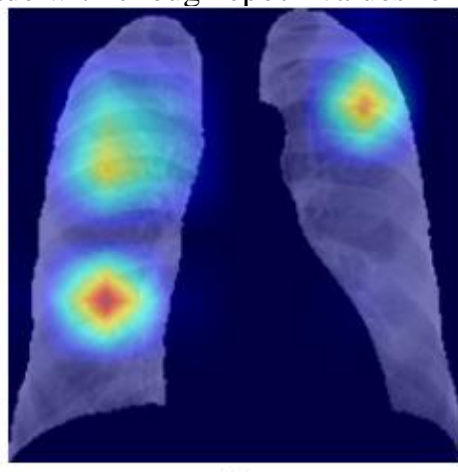

(k)

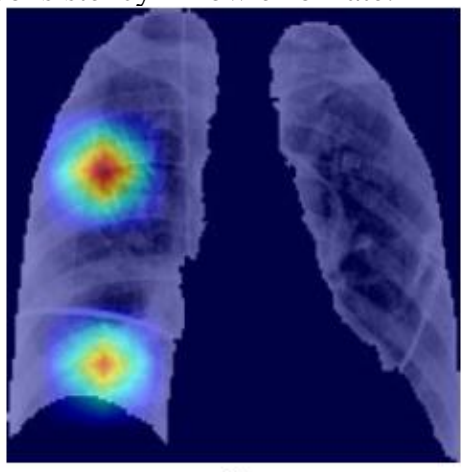

(1)

Figure 8: The final result evaluated on analyzing the dataset.

We've utilized the categorical cross-entropy reduction to instruct model. It's utilised to improve the worth of parameters utilized within the version. We mean to lower losing work together using consecutive epochs. We've utilized adam optimizer using an understanding speed 0.001 to get coaching that our version.

$$
\mathrm{L}\left(\mathrm{Y}, \mathrm{Y}_{1}\right)=\mathrm{Y}^{*} \log \left(\mathrm{Y}_{1}\right)+(1-\mathrm{Y}) * \log \log \left(1-\mathrm{Y}_{1}\right)
$$

Proposed algorithm

$$
\text { Where, } \mathrm{Y}=\text { true label, } \mathrm{Y}_{1}=\text { Predicted Labels, and } \mathrm{L}\left(\mathrm{Y}, \mathrm{Y}_{1}\right) \text { is loss function }
$$

The approach used for implementing the proposed model is discussed below

Step 1:Pre-process image i.e. image $=\mathrm{X}$,

Pre-processing used (We have utilized Keras data generator for this purpose:

i.)Reshape image $(\mathrm{X})$ to $(128,128,3)$

ii.) Random rotation range $=10^{\circ}$

iii.)Horizontal Flip $=$ True

iv.)Zoom Range $=0.4$

Note: shape $=(128,128,3)$ for fast processing

shape $=(256,256,3)$ for better performance

Step 2:Apply the image to an input of the pre-trained model:

Step 3:Fetch the output of the last convolution layer of the given model.

Step 4:Flatten dimensions with reducing $\mathrm{n}$ dimensions to $\mathrm{n}-1$.

Step 5:Apply a dense layer, units $=256$ for XCeption Net and Inception Net

units $=128$ for ResNeXt, where, $\mathrm{W}=$ Weights and $\mathrm{b}=$ bias

Step 6:Apply Activation

$\mathrm{A}=$ LeakyReLU(Z)

Step 7:Apply Dense Layer for inference

$\mathrm{Z}=\mathrm{W} * \mathrm{~A}+\mathrm{b}$
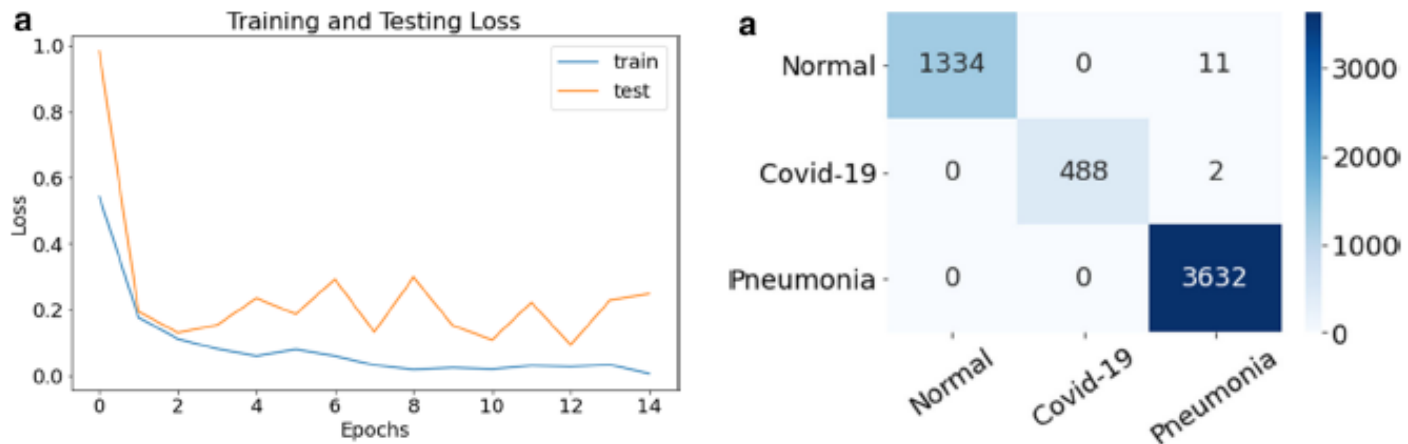

Figure 9: Train and test data with error loss and confusion matrix 

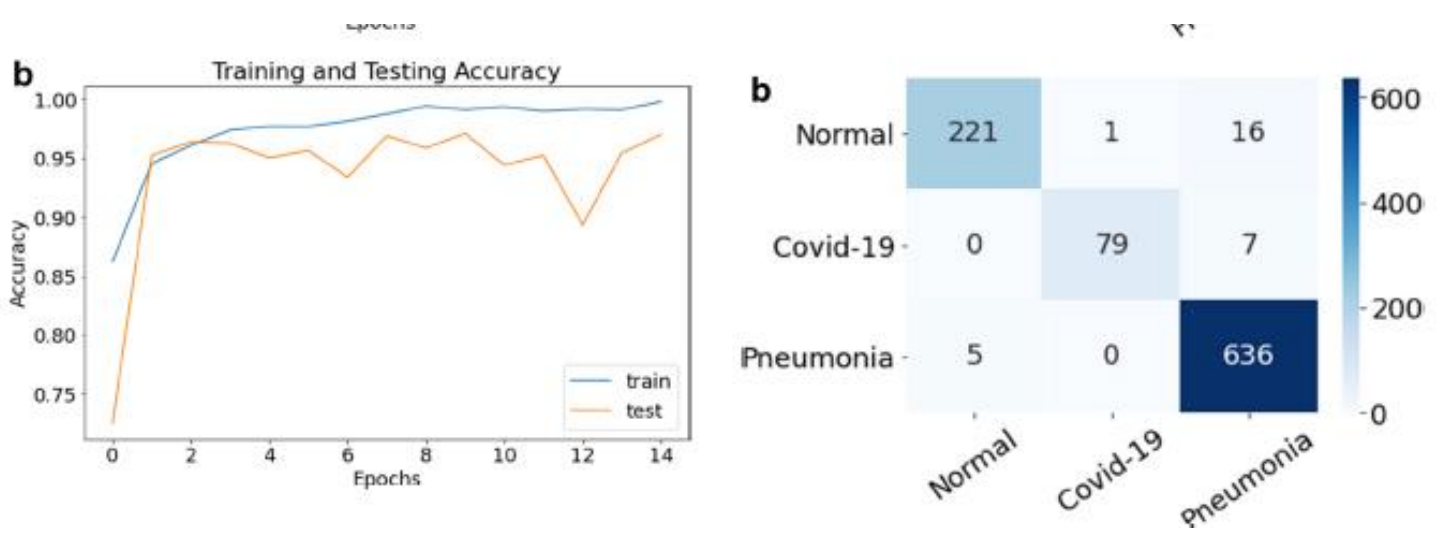

Figure 10: Train and test data with accuracy and confusion matrix

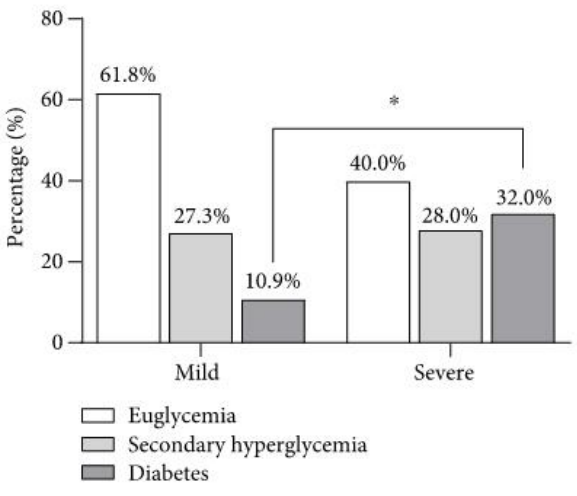

Figure 11: Patients of the severe diabetic type and critical type.

$32 \%$ of COVID-19 acute cases had been patients having diabetes. By comparison, just $10.9 \%$ of moderate instances experienced diabetes (). The corresponding percentages have been shown 78 scenarios $(97.5 \%)$ had signs of pneumonia CT on the list of eighty COVID-19 sufferers, and also the corresponding percentages from the euglycemia category, secondary hyperglycemia category, along with diabetes set ended up $95.5 \%, 100 \%$, and $100.0 \%$, respectively. In addition, the hens were spread at the lungs to get several sufferers (83.8\%), the corresponding percentage from the diabetes collection has been $92.9 \%$, that has been more compared to in both of the other teams. What's more, there have been 6-8 circumstances with ground glass opacities, 24 circumstances with hasty shadows, 26 circumstances with moderate shadows, and 10 circumstances with reticular shadows, and 10 circumstances with fibrosis, 6 circumstances using consolidations, two circumstances with punctate calcification, along with two instances with cerebral effusion. The frequency of assessed CT imaging attributes within three different classes

\section{Conclusion}

COVID-19 can be really actually just a infectious disorder seen as a substantial infectivity and mortality, and specially to patients who have acute underlying disorders like diabetes. Inside this analysis, $17.5 \%$ of COVID-19 sufferers additionally had diabetes, obesity which had been in keeping with all the prior reports where the percentage of COVID-19 sufferers having diabetes ranged from $10.1 \%$ to $20 \%$, and also the ratio of COVID-19 acute scenarios with diabetes has been 32 percent, that had been greater compared to statistics. We've got classified covid-19 scans, also it simplifies the potential extent of employing such processes at the foreseeable future to automate investigation jobs. The top precision accessed might possibly be an effect of problem as it might be due to overfitting. This is sometimes confirmed by analyzing it towards fresh data which was created public fleetingly. Later on, the huge data set for torso xrays is thought to confirm that our suggested version about it. It's likewise advisable to consult with caregivers to get any technical usage the event of the undertaking. We usually do not mean to come up with a ideal discovery mechanism however merely search possible efficiently viable methods to battle 
this particular disorder. Such approaches Could Possibly Be chased to get Additional Studying to Show their actual instance execution.

\section{References:}

[1] Guan WJ, Ni ZY, Hu Y, et al. Clinical characteristics of coronavirus disease 2019 in China. N Engl J Med. 2020;382:1708-1720. doi: 10.1056/NEJMoa2002032. [PMC free article] [PubMed] [CrossRef] [Google Scholar]

[2] Guan WJ, Liang WH, Zhao Y, et al. Comorbidity and its impact on 1590 patients with COVID-19 in China: a nationwide analysis. Eur Respir J. 2020;55:2000547. doi: 10.1183/13993003.00547-2020. [PMC free article] [PubMed] [CrossRef] [Google Scholar]

[3] Singh AK, Gupta R, Ghosh A, Misra A. Diabetes in COVID-19: prevalence, path-ophysiology, prognosis and practical considerations [published online aheadof print, 2020 Apr 9] Diabetes Metab Syndr 2020;14(4):303e10.https://doi.org/10.1016/j.dsx.2020.04.004.

[4] Yang JK, Lin SS, Ji XJ, Guo LM. Binding of SARS coronavirus to its receptor dam-ages islets and causes acute diabetes. Acta Diabetol 2010;47(3):193e9.https://doi.org/10.1007/s00592-009-0109-4.

[5] Schoen K, Horvat N, Guerreiro NFC, de Castro I, de Giassi KS. Spectrum of clin-ical and radiographicfindings in patients with diagnosis of $\mathrm{H} 1 \mathrm{~N} 1$ and correla-tion with clinical severity. BMC Infect Dis 2019;19(1):964.https://doi.org/10.1186/s12879-019-4592-0. Published 2019 Nov 12.

[6] Banik GR, Alqahtani AS, Booy R, Rashid H. Risk factors for severity and mortal-ity in patients with MERSCoV: analysis of publicly available data from SaudiArabia. Virol Sin 2016;31(1):81e4.https://doi.org/10.1007/s12250-015-3679-z.

[7] Zhou J, Tan J. Diabetes patients with COVID-19 need better blood glucosemanagement in Wuhan, China $\begin{array}{lllll}\text { [published online ahead } & \text { of } & 2020\end{array}$ Mar24].Metabolism2020;107:154216.https://doi.org/10.1016/j.metabol.2020.154216.

[8] Geerlings SE, Hoepelman AI. Immune dysfunction in patients with diabetesmellitus (DM). FEMS Immunol Med Microbiol 1999;26(3e4):259e65.https://doi.org/10.1111/j.1574-695X.1999.tb01397.x.

[9] Wang A, Zhao W, Xu Z, Gu J. Timely blood glucose management for theoutbreak of 2019 novel coronavirus disease (COVID-19) is urgently needed[published online ahead of print, 2020 Mar 13]. Diabetes Res Clin Pract2020;162:108118.https://doi.org/10.1016/j.diabres.2020.108118.

[10] Rao S, Lau A, So H-C. Exploring diseases/traits and blood proteins causallyrelated to 284 expression of ACE2, the putative receptor of 2019-nCov: a Men-delian Randomization analysis. Preprint at,https://www.medrxiv.org/content/10.1101/2020.03.04.20031237v1.

[11] Bindom SM, Lazartigues E. The sweeter side of ACE2: physiological evidencefor a role in diabetes. Mol Cell Endocrinol 2009;302(2):193e202.https://doi.org/10.1016/j.mce.2008.09.020.

[12] Guan WJ, Ni ZY, Hu Y, et al. Clinical characteristics of coronavirus disease 2019in China [published online ahead of print, 2020 Feb 28] N Engl J Med 2020.https://doi.org/10.1056/NEJMoa2002032. NEJMoa2002032.

[13] Lause M, Kamboj A, Fernandez Faith E. Dermatologic manifestations of endo-crine disorders. Transl Pediatr 2017;6(4):300e12.https://doi.org/10.21037/tp.2017.09.08.[14] Lin L, Lu L, Cao W, Li T. Hypothesis for potential pathogenesis of SARS-CoV-2infection-a review of immune changes in patients with viral pneumonia.Emerg Microb Infect 2020;9(1):727e32.

[14] Chandra Sekhar Reddy L (2020), "RANKING OF ONLINE FOOD ORDERING AND DELIVERY APPLICATIONS USING DEEP QUEUE LEARNING DATA ANALYTICS IN SOCIAL COMMUNITY ENVIRONMENT“,ISSN 2515-8260, Volume 7, issue-2, pp: 34-3240. 